\title{
Análisis bioeconómico de la pesquería de sardina (Sardinops sagax) y anchoveta (Engraulis ringens) de la zona norte de Chile
}

\author{
Bernardo Aliaga R. ${ }^{1,2}$, Daniel Gómez U. ${ }^{1}$ y Sergio Neira A. ${ }^{1}$ \\ ${ }^{1}$ Programa de Magíster en Pesquerías, Casilla 160-C, Universidad de Concepción. \\ E-mail: dgomez@udec.cl; seneira@udec.cl \\ ${ }^{2}$ Proyecto Ordenamiento Territorial de la Zona Costera de la Región del Bío Bío, \\ Aníbal Pinto 442, Piso 4, Concepción. \\ E-mail: aliaga@zonacostera.cl
}

Recibido: 18 enero 2000; versión corregida: 25 julio 2001; aceptado: 20 agosto 2001

\begin{abstract}
RESUMEN. Se analizó bioeconómicamente la pesquería pelágica industrial de sardina y anchoveta de la zona norte de Chile, integrando información biológico-pesquera (período 1980-1991) y económica (período 1988-1995) mediante el modelo Gordon-Schaefer. Los datos económicos indican que los costos totales de la flota y las plantas de proceso mostraron una tendencia general decreciente durante el período estudiado. En cuanto a las inversiones, es posible identificar dos etapas: de 1988 a 1990 con un moderado aumento de las inversiones, y de 1992 a 1995, con una caída de la inversión neta explicable por la baja en los rendimientos físicos de la producción de harina, la caída en las capturas globales de la pesquería y la caída en el tipo de cambio.

Los puntos de referencia biológicos y económicos determinados con el modelo Gordon-Schaefer, muestran que la flota ha operado utilizando un esfuerzo mayor al correspondiente en el punto de máximo rendimiento económico $\left(E_{M R E}\right)$ y en el punto de equilibrio de libre acceso $\left(E_{L A}\right)$, en tanto que las capturas han sobrepasado el Máximo Rendimiento Sustentable $(M R S)$. Se discuten estos resultados en términos de las limitaciones del modelo teórico al tratar de explicar el comportamiento de la flota en esta pesquería para el período analizado, bajo condiciones de integración vertical en la actividad económica. De resultar efectiva esta hipótesis, modelos bioeconómicos más complejos estarían sujetos a idéntica limitación, al no representar adecuadamente la racionalidad económica integrada de la empresa pesquera industrial. Se proponen futuras líneas de trabajo en esta temática.
\end{abstract}

Palabras claves: bioeconomía, modelo Gordon-Schaefer, pesca industrial, integración vertical, sardina, anchoveta.

\section{Bioeconomic analysis of Pacific sardine (Sardinops sagax) and anchovy (Engraulis ringens) fishery from northern Chile}

\begin{abstract}
The industrial pelagic fishery of pacific sardine and anchovy from northern Chile was analysed through a bioeconomic approach including statistical data from the fishery (catch and effort between 1980 and 1991) and economic data (costs, revenues, investment and employment between 1988 and 1995) using a Gordon-Schaefer model. The economic data allowed us to conclude that costs from the fleet, fishmeal industry and canning industry decreased during the studied period. The financial investment occurred in two different periods. During the first period, investment increased between 1988 and 1991, and during the second period, between 1991 and 1995, investment decreased, which might be explained by a drop in fishmeal yield, catches and money exchange rate.

The biological and economic reference points determined by the Gordon-Schaefer model showed that the fleet has harvested above both the effort in maximum economic yield $\left(E_{M E Y}\right)$ and effort in open access $\left(E_{O A}\right)$, while catches have exceeded the Maximum Sustainable Yield $(M S Y)$. These results are discussed in terms of the theoretical model's limitations to explain the functioning of the pelagic fishery fleet under backward-forward integration. It is hypothesized that more complex bioeconomic models would be subjected to identical limitation, because they would inadequately represent the integrated economic rationality of the industrial fishery. Some future worklines on the same topic are proposed.
\end{abstract}

Key words: bioeconomics, Gordon-Schaefer model, industrial fishery, backward-forward integration, pacific sardine, anchovy. 


\section{INTRODUCCIÓN}

En la zona norte de Chile (I y II Regiones) existe desde fines de la década del 50 una pesquería pelágica industrial orientada a la captura y proceso de sardina (Sardinops sagax) y anchoveta (Engraulis ringens). Ambas especies se caracterizan por ser recursos costeros, presentar un rápido crecimiento, ciclo de vida corto y elevada tasa de mortalidad natural, siendo su distribución espacial, biomasa y reclutamiento fuertemente influenciados por factores ambientales (Serra, 1983; Cubillos, 1991).

La pesquería se basa en sus inicios en la anchoveta y tiene su primera crisis importante en 1965, coincidiendo con un fenómeno de El Niño intenso. A partir de ese año se produce una brusca caída en las capturas y reducción en el stock parental, colapsando la pesquería en 1973 tanto en Chile como en Perú (SUBPESCA, 1996). La captura de sardina se inicia en 1976, tres años después del colapso de la pesquería de anchoveta. El desembarque de sardina crece desde 1976 hasta 1985, alcanzando un máximo histórico de 2,6 millones de toneladas en 1985, y luego declina a partir de 1986, con un mínimo de 9 mil toneladas en 1998 (SUBPESCA, 1996; SERNAPESCA, 2000) (Fig. 1).

A partir de 1985 se evidencian los primeros síntomas de recuperación del stock de anchoveta, como consecuencia de reclutamientos fuertes. Esta recuperación coincide con la disminución en la abundancia de sardina, registrándose un desembarque creciente de anchoveta que alcanza a 2,1 millones de toneladas en 1994 (SUBPESCA, 1996; SERNAPESCA, 2000).

La sobreexplotación de los recursos pesqueros, sumada a la sobrecapitalización de las pesquerías, ha motivado la utilización creciente de herramientas económicas para su estudio. De esta forma, la administración pesquera actual requiere un análisis integral no sólo de los factores biológicos y ecológicos que determinan la dinámica de un stock, sino también de los agentes económicos involucrados en la actividad pesquera (Barber y Taylor, 1990). De aquí que las herramientas bioeconómicas se apliquen en forma creciente en el ámbito pesquero, integrando información económica con aquella propia del stock explotado (Sinclair et al., 1997; Cerda et al., 1997).

Un modelo bioeconómico básico aplicado a pesquerías es el modelo Gordon-Schaefer, derivado de los trabajos de Gordon (1953, 1954) y Schaefer (1954). Este modelo, que resulta extremadamente simple, clarifica los problemas básicos de una pesquería, sirve como un instrumento didáctico y ha demostrado su utilidad al confirmarse la validez de algunas de sus conclusiones mediante el uso de modelos más complejos (Jiles y Calfucura, 1994).

Tomando en cuenta estos antecedentes y la escasa literatura nacional en bioeconomía aplicada a pesquerías industriales nacionales (Cerda et al., 1997), el objetivo de este trabajo es analizar la pesquería pelágica industrial de sardina y anchoveta de la I y II Regiones mediante instrumentos bioeconómicos, de manera de comprender aspectos económicos de su estructura y funcionamiento.

\section{MATERIALES Y MÉTODOS}

\section{Antecedentes económicos de la pesquería}

La información económica básica para realizar este trabajo se obtuvo de la Encuesta Nacional de la Industria Manufacturera (ENIA) y la Encuesta de Flota Propia (EFP), ambas realizadas

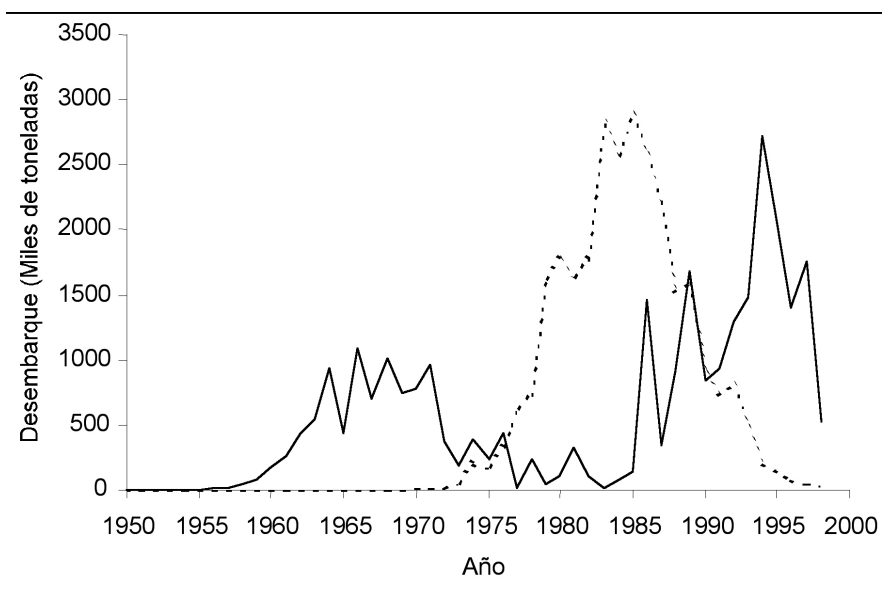

Figura 1. Desembarque histórico (1950-1998) de anchoveta (línea continua) y sardina (línea punteada) en la costa de Chile (Fuente: FishBase, 1998; SERNAPESCA, 2000).

Figure 1. Historical landings (1950-1998) of anchovy (solid line) and pacific sardine (dotted line) in the coast of Chile (Source: FishBase, 1998; SERNAPESCA, 2000). 
anualmente por el Instituto Nacional de Estadísticas de Chile (INE). ENIA contiene tanto información de plantas reductoras (código CIIU 3115) como elaboradoras de conservas (código CIIU 3114). EFP se aplica a los establecimientos manufactureros que se autoabastecen de materia prima; en este caso, la flota también recibe un código (CIIU 1301). La serie de tiempo empleada en este estudio abarcó desde 1988 hasta 1995. La información contiene las variables costos, ingresos, inversiones y empleo.

Los costos totales en la industria reductora (i.e. harina) y conservera fueron proporcionados directamente en la encuesta. En el caso de la flota, fueron estimados desde el salario fijo y variable (primas de pesca), los costos intermedios (combustible e insumos) y la depreciación. Como una medida proxy de los ingresos en los tres sectores se obtuvo el Valor Bruto de la Producción (VBP), directamente de la encuesta. En el caso de la flota, el VBP se estimó a partir del valor de la captura destinada a establecimientos propios y de terceros, informada directamente en la encuesta.

La inversión en la flota consideró el cálculo de la inversión bruta y neta (i.e. inversión bruta menos la depreciación). Por otra parte, el empleo en los tres sectores fue cuantificado mediante la ocupación media del trabajo.

En todos los casos, las series de tiempo con datos económicos se trabajaron en términos reales, usando un deflactor construido especialmente para el sector pesquero.

\section{Modelo bioeconómico Gordon-Schaefer}

\section{Submodelo biológico (Schaefer)}

La información de captura y esfuerzo de la pesquería industrial de sardina y anchoveta se obtuvo desde la literatura para una serie de tiempo que abarca de 1980 a 1991 (Tabla 1) (Caballero et al., 1992). La unidad de esfuerzo utilizada fue viajes con pesca total de recursos sardina y anchoveta (VPT), considerada la más adecuada para esta pesquería (Caballero et al., 1992). El barco patrón determinado corresponde a un barco de $140 \mathrm{~m}^{3}$ de capacidad de bodega, representativo de la flota pelágica industrial de la zona norte de Chile en el período de análisis.

El modelo de producción global de Schaefer (1954), expresado como ecuación de diferencias de acuerdo a Hilborn y Walters (1992), puede escribirse como

$$
\mathrm{B}_{\mathrm{t}+1}=\mathrm{B}_{\mathrm{t}}+\mathrm{r} * \mathrm{~B}_{\mathrm{t}} *\left(1-\frac{\mathrm{B}_{\mathrm{t}}}{\mathrm{K}}\right)-\mathrm{C}_{\mathrm{t}}
$$

la que permite predecir la biomasa remanente del stock como resultado de la captura $\left(C_{t}\right)$, el excedente productivo de crecimiento (segundo término) y la biomasa previa $\left(B_{t}\right)$. El excedente productivo es a su vez función de la biomasa y de dos parámetros de la población: la tasa intrínseca de crecimiento $r$ y el tamaño del stock inexplotado en equilibrio o capacidad de carga del ecosistema $K$ (Hilborn y Walters, 1992; Seijo et al., 1997). La captura se asume proporcional a la biomasa $B$ y esfuerzo de pesca $E$, a través del coeficiente de capturabilidad $q$

$$
\mathrm{C}_{\mathrm{t}}=\mathrm{q} * \mathrm{E}_{\mathrm{t}} * \mathrm{~B}_{\mathrm{t}}
$$

Bajo el supuesto que la captura remueve sólo el excedente productivo (i.e. $B_{t+1}=B_{t}$ ) y combinando las ecuaciones (1) y (2), el modelo se reduce a

$$
\mathrm{C}_{\mathrm{t}}=\mathrm{q} * \mathrm{~K} * \mathrm{E}_{\mathrm{t}} *\left(1-\frac{\mathrm{q} * \mathrm{E}_{\mathrm{t}}}{\mathrm{r}}\right)
$$

Tabla 1. Datos históricos de captura (en toneladas) y esfuerzo (VPT, Viajes con pesca total) para los recursos sardina y anchoveta utilizada en el modelo bieconómico Gordon-Schaefer (Caballero et al., 1992).

Table 1. Historical data of catch (in tonnes) and effort (Total fishing trips) of both the species pacific sardine and anchovy utilised in the GordonSchaefer bioeconomic model (Caballero et al., 1992).

\begin{tabular}{|ccc|}
\hline AÑO & $\begin{array}{c}\text { CAPTURA } \\
\text { (toneladas) }\end{array}$ & $\begin{array}{c}\text { ESFUERZO } \\
\text { (Viajes con pesca total) }\end{array}$ \\
\hline 1980 & 2043460 & 14541 \\
1981 & 2146239 & 19331 \\
1982 & 2453678 & 18698 \\
1983 & 2637244 & 21162 \\
1984 & 3036685 & 20894 \\
1985 & 3092055 & 19932 \\
1986 & 3354273 & 24132 \\
1987 & 2240475 & 20191 \\
1988 & 2352229 & 19055 \\
1989 & 2904644 & 21854 \\
1990 & 1645615 & 15823 \\
1991 & 1557268 & 13570 \\
& & \\
\hline
\end{tabular}


A partir de la ecuación (3) los parámetros $K$ y $r$ se estiman mediante mínimos cuadrados lineales. Para ello, se utiliza un valor del coeficiente de capturabilidad $q$ para la pesquería obtenido de Agüero y González (1996), único para la pesquería conjunta de sardina y anchoveta.

Por otro lado, reemplazando la ecuación (2) en la (3), podemos simular la biomasa sustentable (i.e. en equilibrio) como función del esfuerzo (Seijo et al., 1997)

$$
\mathrm{B}_{\mathrm{t}}=\left(1-\frac{\mathrm{q} * \mathrm{E}_{\mathrm{t}}}{\mathrm{r}}\right) * \mathrm{~K}
$$

\section{Submodelo económico (Gordon)}

Gordon (1954) estableció que la función de ingresos netos p en una pesquería de acceso abierto puede expresarse matemáticamente como la diferencia entre los ingresos totales sostenibles (ITS) y los costos totales $(C T)$, que son funciones de la captura $C$ y esfuerzo $E$ respectivamente:

$$
\pi=\mathrm{ITS}-\mathrm{CT}=\mathrm{p} * \mathrm{C}-\mathrm{a} * \mathrm{E}
$$

En la ecuación anterior $p$ es el precio de la captura unitaria $(\$ / \mathrm{t})$ y $a$ es el costo unitario del esfuerzo (\$/VPT). Para modelar la captura en términos económicos y los costos totales en equilibrio, se utilizó la ecuación (3) y los datos de esfuerzo. Se utilizaron los precios de venta de la captura a terceros y el costo unitario promedio del esfuerzo del año 1989, que fue definido como año de referencia, para homogeneizar los precios en el análisis económico.

Una vez determinado el modelo GordonSchaefer (G-S) para la pesquería, se calcularon los puntos de referencia bioeconómicos de interés para la administración de una pesquería (Tabla 2).

Para determinar la captura y la biomasa en los puntos de máximo rendimiento y rendimiento de libre acceso, se utilizaron los valores de esfuerzo de la Tabla 3 y se reemplazaron en las ecuaciones (3) y (4).

\section{RESULTADOS}

\section{Antecedentes económicos}

\section{Costos e ingresos}

Los costos totales e ingresos (Valor Bruto de la Producción, VBP) de la pesquería pelágica industrial del norte de Chile, y su desagregación en los sectores flota, harina y conservas durante el período analizado, siguen una tendencia decreciente hacia el año 1995 (Figs. 2 y 3). En 1993, los costos totales de operación en la flota superaron el VBP (Fig. 3). En los sectores de producción de harina y de conservas el VBP fue superior a los costos totales.

En cuanto a los ingresos, el análisis sectorial del VBP muestra que la industria reductora participa en más del 70\% de los ingresos, seguida en importancia por la actividad económica generada en la flota $\mathrm{y}$, en último lugar, por la industria conservera (Fig. 4).

\section{Inversiones y empleo}

Las inversiones brutas en la flota entre 1988 y 1995 fueron en aumento sostenido a partir de 1988 y hasta 1990. Durante 1991 no se realizaron inversiones en flota en esta pesquería (Fig. 5). En 1992, la inversión en flota superó los 6 mil millones de pesos, y luego disminuyó en los años siguientes. El descenso repentino en las inversiones en flota en 1991, puede ser explicado por el ambiente de inestabili-

Tabla 2. Puntos de referencia bioeconómicos de interés para la administración de una pesquería (extraído y modificado de Hilborn y Walters, 1992 y Seijo et al., 1997).

Table 2. Bioeconomic reference points useful for fishery management (extracted and modified from Hilborn and Walters, 1992 and Seijo et al., 1997).

\begin{tabular}{|lc|}
\hline \multicolumn{1}{|c|}{ Parámetro } & Ecuación \\
\hline Esfuerzo de pesca en el punto de Máximo Rendimiento Sostenible $\left(\mathrm{E}_{\mathrm{MRS}}\right)$ & $E_{M R S}=r / 2 q$ \\
Máximo Rendimiento Sostenible (MRS) & $M R S=r K / 4$ \\
Biomasa en el punto de Máximo Rendimiento Sostenible (B $\mathrm{MRS})$ & $B_{M R S}=K / 2$ \\
Esfuerzo de Máximo Rendimiento Económico (máx [ITS-CT]) $\left(\mathrm{E}_{\mathrm{MRE}}\right)$ & $E_{M R E}=(r / 2 q)(1-[a / p q K])$ \\
Esfuerzo en Equilibrio Bioeconómico o Libre Acceso $(I T S=C T)\left(\mathrm{E}_{\mathrm{EBE}}\right.$ o $\left.\mathrm{E}_{\mathrm{LA}}\right)$ & $E_{E B E}=E_{L A}=(r / q)(1-[a / p q K])$ \\
\hline
\end{tabular}


dad provocado por la discusión en el Parlamento de Chile de la Ley General de Pesca y Acuicultura, aprobada en septiembre de 1991. La posterior declinación de las inversiones en la pesquería pelágica del norte se explica por un conjunto de factores, entre los cuales está la baja en los rendimientos físicos de la producción de harina, la caída en las capturas globales de la pesquería y la caída en el tipo de cambio.

Los datos de ocupación media del trabajo permiten aseverar que los dos sectores más importantes de la pesquería pelágica industrial de la zona norte, en cuanto al empleo total que generan, son la flota y la industria reductora (Fig. 6).

\section{Modelo bioeconómico Gordon- \\ Schaefer}

El modelo de regresión ajustado a los datos de captura y esfuerzo de la pesquería de sardina y anchoveta entre 1980 y 1991 mostró una correlación significativa $\left(\mathrm{R}^{2}=0,75 ; \mathrm{P}<0,001 ; \mathrm{n}=12\right)$. Los valores obtenidos para los parámetros biológicos del submodelo biológico fueron $K=4,3$ millones de t y $r=1,15$ año $^{-1}$.

Los valores del precio unitario de la captura y el costo unitario del esfuerzo en 1989 fueron $p=\$ 46300 / \mathrm{t}$ y $a=$ $\$ 2$ millones/VPT, respectivamente. Con estos datos, se construyó la curva de ITS y $C T$ del modelo G-S, con los puntos de referencia bioeconómicos (Fig. 7). La biomasa (en equilibrio) se observa en la Fig. 8, al igual que los valores esperados de biomasa cuando el esfuerzo coincide con los puntos de referencia bioeconómicos (Tabla 3).

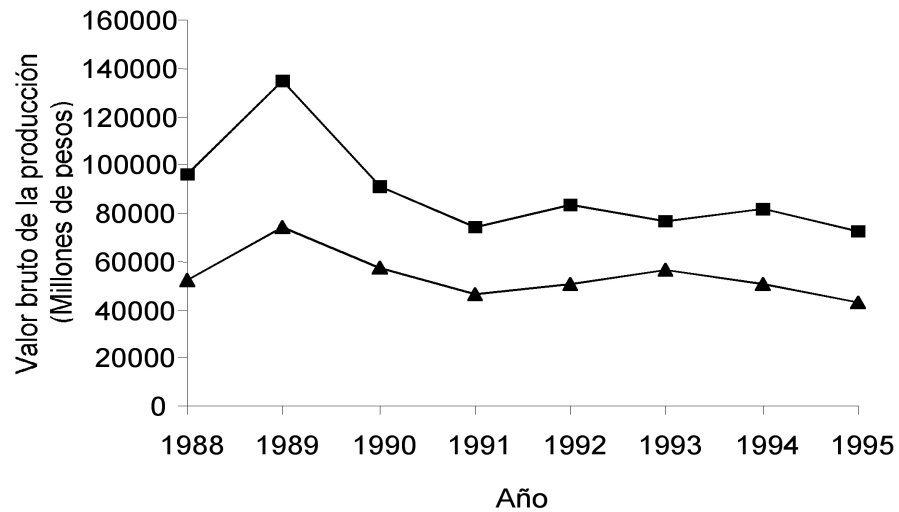

Figura 2. Valor bruto de la producción (cuadrados) y costos totales (triángulos) de la pesquería pelágica del norte de Chile entre 1988 y 1995.

Figure 2. Gross production value (squares) and total costs (triangles) of the pelagic fishery from northern Chile between 1988 and 1995.

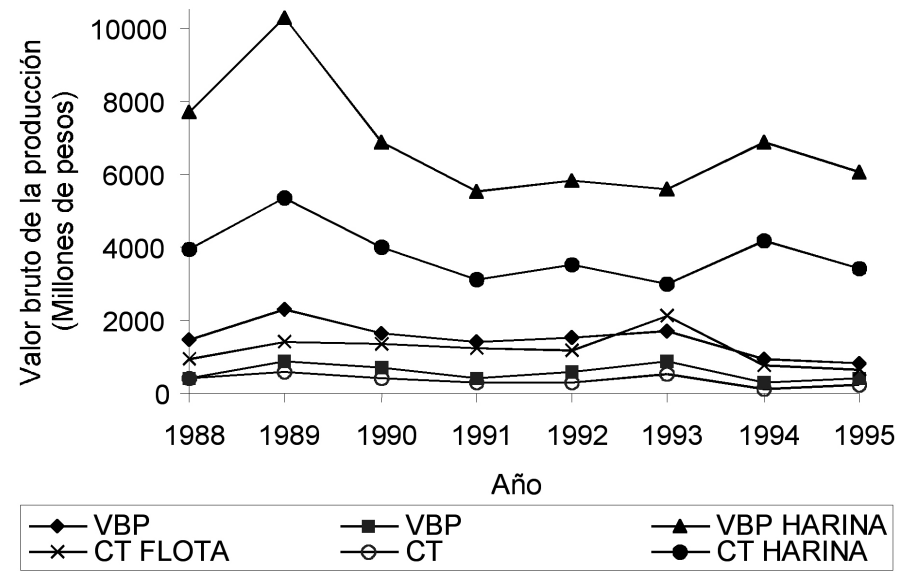

Figura 3. Valor bruto de la producción y costos totales por sector de la pesquería pelágica del norte de Chile entre 1988 y 1995.

Figure 3. Gross production value and total costs per sector of the pelagic fishery from northern Chile between 1988 and 1995.

Tabla 3. Valores de los puntos de referencia bioeconómicos de la pesquería pelágica industrial del norte de Chile. Table 3. Values of the bioeconomic reference points of the pelagic fishery from northern Chile.

\begin{tabular}{|lcccc|}
\hline & $\begin{array}{c}\text { CAPTURA } \\
\text { (millones de t) }\end{array}$ & $\begin{array}{c}\text { INGRESOS } \\
\text { (millones de \$) }\end{array}$ & $\begin{array}{c}\text { ESFUERZO } \\
\text { (VPT) }\end{array}$ & $\begin{array}{c}\text { BIOMASA } \\
\text { (millones de t) }\end{array}$ \\
\hline Máximo Rendimiento Económico (MRE) & 0,511 & 23715,5 & 5776 & 3,788 \\
Rendimiento de Libre Acceso (RLA) & 0,897 & 41561,8 & 11552 & 3,319 \\
Máximo Rendimiento Sostenible (MRS) & 1,228 & 56895,7 & 26226 & 2,128 \\
\hline
\end{tabular}




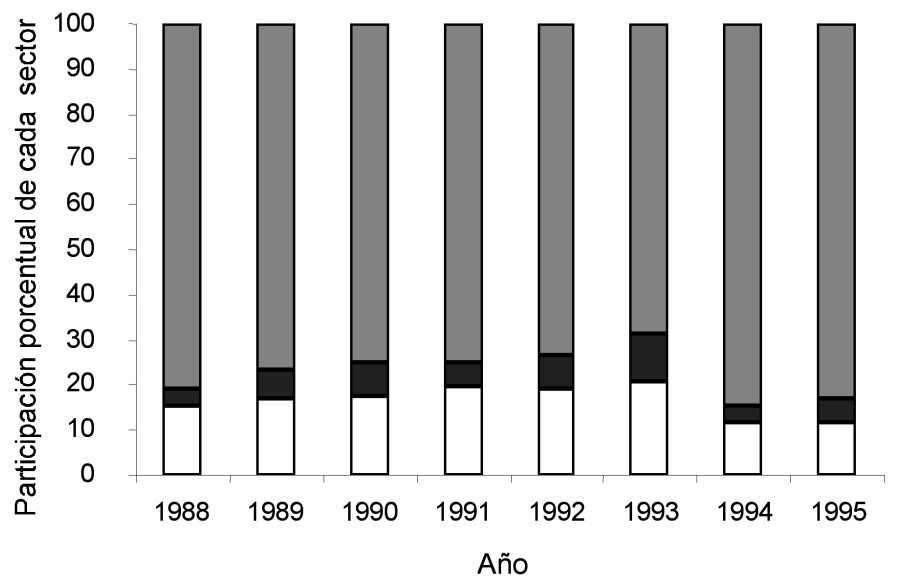

Figura 4. Participación porcentual de cada sector en el valor bruto de la producción de la pesquería pelágica del norte de Chile entre 1988 y 1995 (barras grises: industria reductora; barras negras: industria conservera; barras blancas: flota).

Figure 4. Percentage of each sector in the gross production value of the pelagic fishery from northern Chile between 1988 and 1995 (gray bars: fishmeal industry; black bars: canning industry; white bars: fleet).

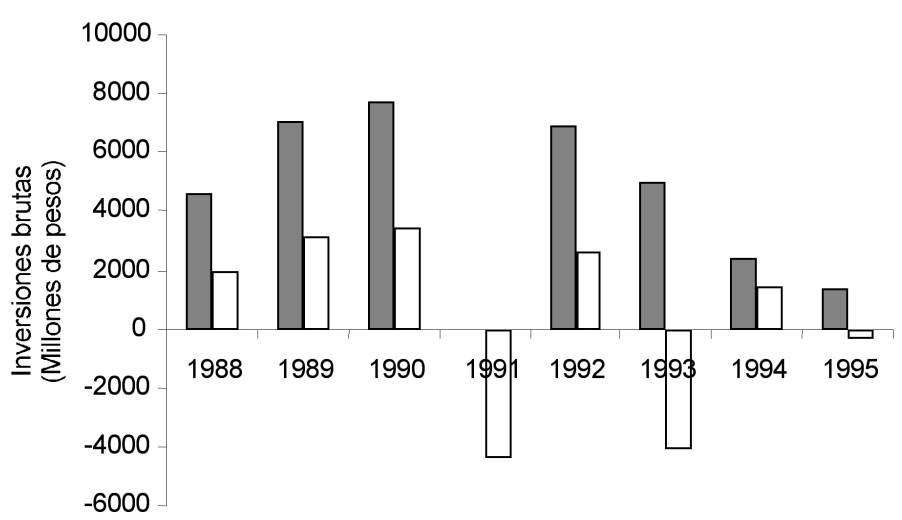

Año

Figura 5. Inversiones brutas (barras oscuras) y netas (barras claras) de la flota en la pesquería pelágica del norte de Chile entre 1988 y 1995.

Figure 5. Gross investments (dark bars) and net investments (light bars) in fleet of the pelagic fishery from northern Chile between 1988 and 1995.

En la Tabla 3 y Fig. 7 se observa que la captura correspondiente al Rendimiento de Libre Acceso (RLA) es de menor valor que el Máximo Rendimiento Sostenible (MRS). Este es un resultado poco usual, ya que los ejemplos tradicionales ubican siem- pre el RLA por sobre el MRS (Seijo et al., 1997). Aunque de las especificaciones del modelo se deduce que teóricamente es posible que se presente este caso (la pendiente de la curva de CT no está restringida en el modelo), su demostración empírica tiene algunas consecuencias que son objeto de discusión.

\section{DISCUSIÓN}

El modelo G-S ha sido ampliamente aplicado a pesquerías marinas siendo su principal mérito el clarificar en términos económicos el concepto de sobrepesca en pesquerías no reguladas (Seijo et al., 1997). Sin embargo, muchos de los supuestos biológicos y económicos del modelo presuponen un comportamiento ideal tanto del recurso como del sector productivo y el mercado, los que en sentido estricto no serían aplicables a la pesquería en estudio.

La primera limitación de nuestro modelo es asumir que la pesquería de sardina y anchoveta puede ser tratada como una pesquería monoespecífica, cuando en realidad no lo es. Lo anterior implica la estimación de parámetros biológicos de abundancia asintótica $K$ y tasa intrínseca de crecimiento $r$ comunes para ambas especies. De acuerdo con Hilborn y Walters (1992), es posible aplicar modelos de dinámica de biomasa a pesquerías multiespecíficas, especialmente en aquellas en que los datos de captura es difícil o imposible de obtener por separado. En este sentido, analizar las pesquerías por separado es actualmente imposible ya que tanto la información de captura y esfuerzo (Tabla 1) como la base de datos económicos (la base Flota del INE) no está separada por especie.

La segunda limitación se relaciona con el supuesto de equilibrio. En el modelo G-S la variable explícita es el esfuerzo pesquero. En este trabajo en particular, el esfuerzo lleva implícita la va- 


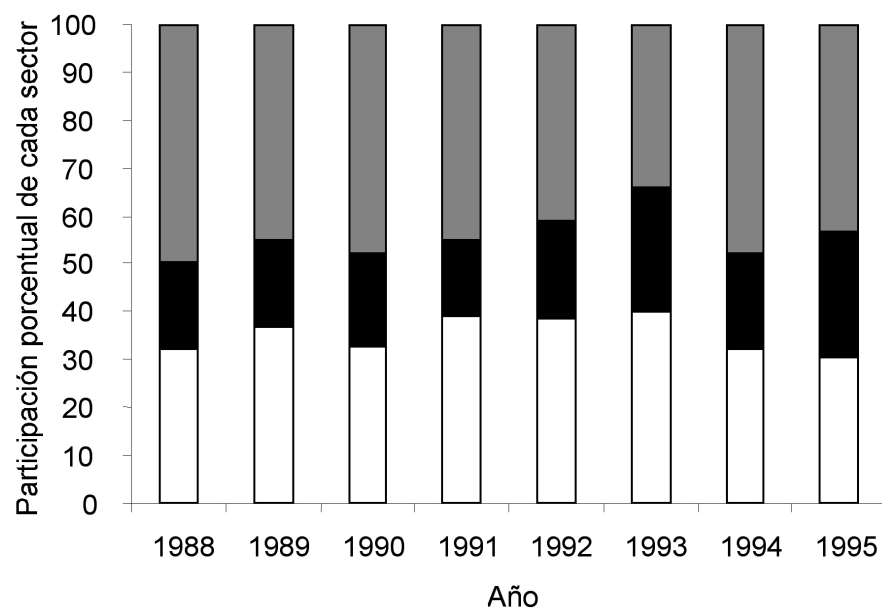

Figura 6. Participación porcentual de cada sector en el empleo en la pesquería pelágica del norte de Chile entre 1988 y 1995 (barras grises: industria reductora; barras negras: industria conservera; barras blancas: flota). Valores de Referencia: 1988: 7802 empleos; 1995: 3986 empleos.

Figure 6. Percentage of each sector in the employment of the pelagic fishery from northern Chile between 1988 and 1995 (gray bars: fishmeal industry; black bars: canning industry; white bars: fleet). Reference values: 1988: 7802 employments; 1995: 3986 employments).

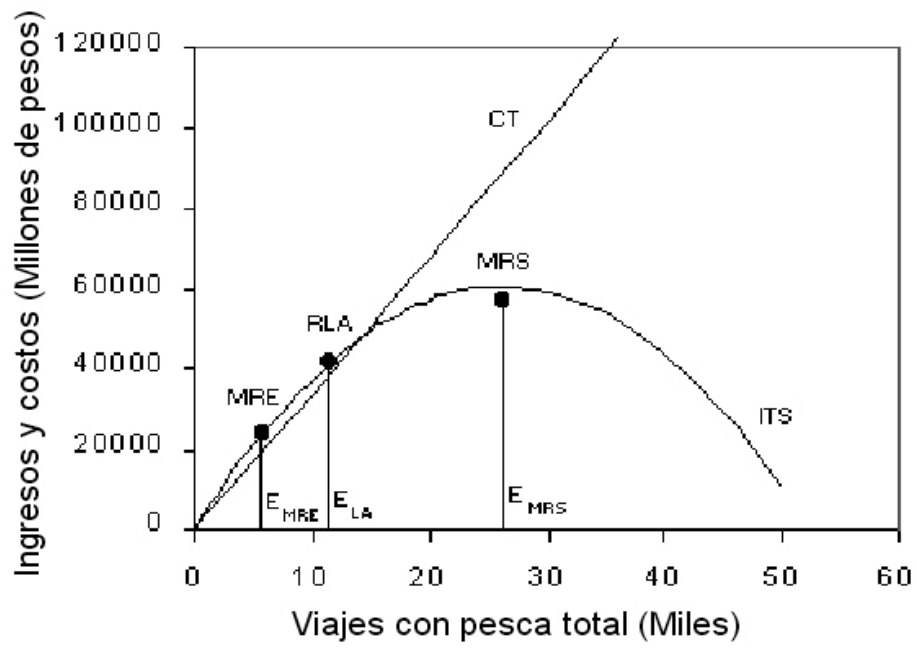

Figura 7. Modelo bioeconómico Gordon-Schaefer para la pesquería pelágica del norte de Chile.

Figure 7. Gordon-Schaefer bioeconomic model of the pelagic fishery from northern Chile.

riable temporal que corresponde a una serie de 11 años. Por lo tanto, el hecho que las series de captura y esfuerzo (Tabla 1) y las series de ingreso y costos de extracción y de proceso se encuentren desfasadas no viola el supuesto de equilibrio (Seijo et al., 1997).
Dado que el objetivo de este trabajo no ha sido abordar las limitaciones propias de la aproximación estática que supone el modelo G-S, ya señalada por otros autores (Clark, 1990), sino que identificar las variables económicas que explicarían el comportamiento de la pesquería de sardina y anchoveta en la zona norte, se considera que las limitaciones anteriores serían una fuente de error adicional e independiente del error de no considerar la integración vertical de la industria.

Al comparar la captura y desembarque efectivos de la pesquería (Fig. 1, Tabla 1), en especial entre 1980 y 1998, con la captura que el modelo predice para cada punto de referencia de la Tabla 3 , se observa que la flota ha capturado ambas especies muy por encima de los mismos. Esto implica que el sector extractivo habría operado con pérdidas económicas, lo que es coincidente con los datos económicos reales (Fig. 3, año 1993), e indica que su actividad compromete la sustentabilidad del stock de sardina y anchoveta, ya que ha aplicado un esfuerzo cercano al que corresponde en el punto de máximo rendimiento sustentable $\left(E_{M R S}\right)$ y mayor al esfuerzo aplicable en el punto de equilibrio de libre acceso $\left(E_{L A}\right)$ y de máximo rendimiento económico $\left(E_{M R E}\right)$.

La operación de la flota con bajos márgenes de ganancia, incluso con pérdidas en 1993, puede explicarse en tanto la industria considerada como un todo siga obteniendo ganancias, mediante la integración vertical de las unidades de planta de proceso y flota, al interior de un mismo grupo empresarial. Dicho de otro modo, la flota transfiere sus pérdidas a la planta al operar en un esquema de integración vertical, como ocurre en el caso de la pesquería industrial pelágica del norte de Chile.

Estos resultados indican que el modelo teórico falla en explicar el comportamiento de la flota en esta pesquería para el período analizado. En efecto, en el punto de equilibrio de libre acceso, 


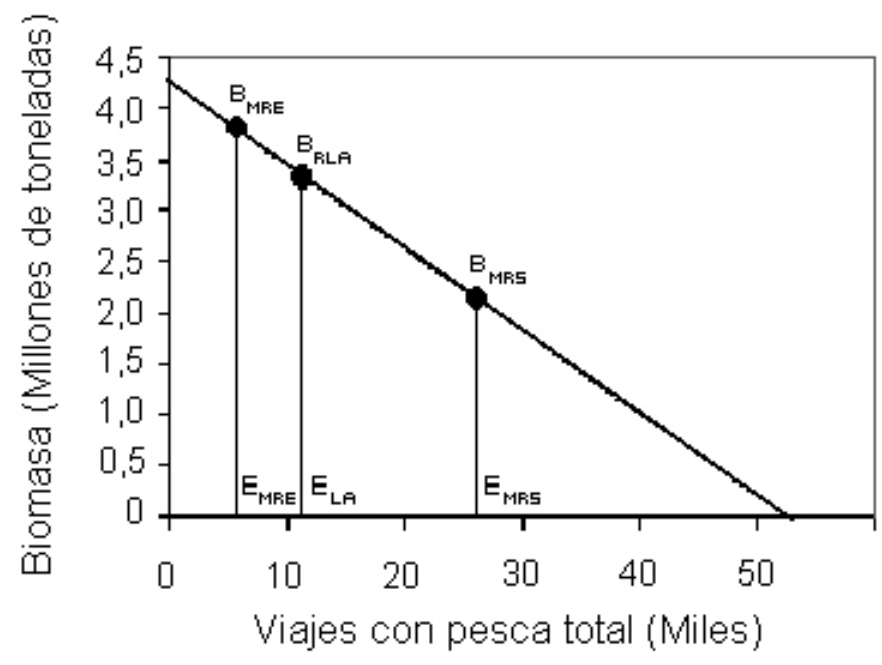

Figura 8. Biomasa sustentable del stock de sardina y anchoveta en función del esfuerzo y parámetros bioeconómicos.

Figure 8. Sustainable biomass of the Pacific sardine and anchovy stock as a function of the effort and bioeconomic reference points.

en que no hay ganancias porque los costos totales se igualan a los ingresos totales, el modelo teórico predice que ya no existen incentivos para incorporar nuevas naves o aumentar el esfuerzo de las naves existentes. En la pesquería pelágica del norte, la flota pesca ejerciendo esfuerzos superiores al del punto de equilibrio de libre acceso, con costos mayores a sus ingresos. Se postula la hipótesis de que la flota ejerce esfuerzos superiores al del equilibrio de libre acceso cuando existe integración vertical, dado que en el proceso de manufactura la industria recupera las pérdidas generadas en la fase extractiva. In extremis, el comportamiento de la flota no tendría ninguna relación con los Puntos de Referencia Bioeconómicos en presencia de integración vertical: la conducta de la flota dependería de las decisiones económicas de la empresa verticalmente integrada.

De resultar efectiva esta hipótesis los modelos más sofisticados, que han incorporado variabilidad en el tiempo (Clark, 1990), o que suponen no-linealidad de la tasa de captura (Amundsen et al., 1995), estarían sujetos a idéntica limitación, al no representar adecuadamente la racionalidad económica integrada de la empresa pesquera industrial.

Considerando que las principales industrias pesqueras nacionales presentan diferentes grados de integración vertical, y que los modelos bioeconómicos hasta ahora realizados para algunas pesquerías no han dado cuenta de los efectos de la integración vertical en la conducta de la flota (Cerda et al., 1997), se identifican tres posibles líneas de trabajo futuro: a) un análisis teórico acerca de los efectos de la integración vertical en el comportamiento del esfuerzo de la flota, b) un análisis teórico acer- ca del funcionamiento de los mercados de productos pesqueros en presencia de integración vertical y c) una revisión del grado de integración vertical de la industria pesquera nacional.

\section{AGRADECIMIENTOS}

Los autores desean agradecer la colaboración de la Universidad de Concepción, que a través de la Escuela de Graduados adquirió la Base de Datos del Instituto Nacional de Estadísticas utilizada en este artículo. Los comentarios de los docentes Renato Quiñones del Departamento de Oceanografía, Facultad de Ciencias Naturales y Oceanográficas, Felipe Vásquez, Miguel Angel Quiroga y Hugo Salgado, del Departamento de Economía, Facultad de Ciencias Económicas y Administrativas de la Universidad de Concepción, y de cuatro revisores anónimos, que permitieron mejorar este documento.

\section{REFERENCIAS}

Agüero, M. y E. González. 1996. Managing transboundary stocks of small pelagic fish. problems and options. World Bank Discussion Paper No. 329 (Fisheries Series). The World Bank, Washington D.C., 40 pp.

Amundsen, E.S., T. Björndal y J.M. Conrad. 1995. Open access harvesting of the Northeast Atlantic minke whale. Environ. Res. Econ., 6: 167-185.

Barber, W. y J. Taylor. 1990. The importance of goals, objectives and values in the fisheries management process and organization: A review. North Amer. J. Fish. Manage., 10(4): 365-373.

Caballero, L., L. Santillan y G. Rosson. 1992. Investigación del esfuerzo pesquero en las pesquerías chilenas pelágicas. Instituto de Fomento Pesquero, Chile, 44 pp. 
Caddy, J. y R. Mahon. 1996. Puntos de referencia para la ordenación pesquera. FAO Doc. Téc. Pesca, 347: 1-109.

Cerda, A., B. Aliaga, R. Dresner, E. Bobenrieth, R. Quiñones, A. Sepúlveda, M. Gálvez y A. Hernández. 1997. Evaluación bioeconómica de la pesquería industrial de jurel en la zona centro-sur. Fase I. Informe Final. Proyecto Fondo de Investigación Pesquera, FIP 95-12, 228 pp.

Clark, C.W. 1990. Mathematical Bioeconomics: The optimal management of renewable resources. John Wiley and Sons, New York. 291 pp.

Cubillos, L. 1991. Estimación mensual de la biomasa, reclutamiento y mortalidad por pesca de la anchoveta (Engraulis ringens) de la zona norte de Chile en el período 1986-1989. Biol. Pesq., 20: 49-59.

FishBase. 1998. FishBase 98. CD-ROM, ICLARM, Manila.

Gordon, H. 1953. An economic approach to the optimum utilization of fishery resources. J. Fish. Res. Bd. Can., 10: 442-457.

Gordon, H. 1954. The economic theory of a common property: The fishery. J. Political Econ., 62(2): 124142.

Hilborn, R. y C. Walters. 1992. Quantitative fisheries stock assessment: Choice, dynamics and uncertainty. Routledge, Chapman y Hall, New York, $570 \mathrm{pp}$.
Jiles, J. y E. Calfucura. 1994. La regulación pesquera chilena en pesquerías pelágicas. En: E. Figueroa (ed.). Políticas económicas para el desarrollo sustentable de Chile. Centro de Recursos Naturales y Medio Ambiente. Universidad de Chile. pp. 205-247.

Schaefer, M. 1954. Some aspects of the dynamics of populations important to the management of the commercial marine fisheries. Bull. Inter-Amer. Trop. Tuna Comm., 1(2): 27-56.

Seijo, J., O. Defeo y S. Salas. 1997. Bioeconomía pesquera. Teoría, modelación y manejo. FAO Doc. Téc. Pesca, 368: 1-176.

Servicio Nacional de Pesca (SERNAPESCA). 2000. Anuario estadístico de pesca 1999. Servicio Nacional de Pesca, Valparaíso, 283 pp.

Serra, R. 1983. Changes in the abundance of pelagic resources along the Chilean coast. FAO Fish. Rep., 291(2): 255-284.

Sinclair M., R. O’Boyle, D. Burke y G. Peacock. 1997. Why do some fisheries survive and other collapse? En: D. Hancock, D. Smith, A. Grant y J. Beumer (eds.). Developing and sustaining world fisheries resources: The state of science and management. Second World Fisheries Congress, Brisbane 1996. CSIRO publishing, Melbourne, pp. 23-35.

Subsecretaría de Pesca (SUBPESCA). 1996. Chile Azul. Principales recursos pesqueros. Subsecretaría de Pesca, Valparaíso, 89 pp. 